\title{
Desenvolvimento de um sistema piloto de informações geográficas para a gestão da piscicultura continental catarinense
}

\author{
Natalia Costa Marchiori'1, André Luís Tortato Novaes² e Bruno Corrêa da Silva ${ }^{3}$
}

\begin{abstract}
Resumo - Apesar do seu crescimento constante nos últimos anos, a piscicultura catarinense se desenvolve em meio a diversos entraves, como a desorganização da cadeia produtiva, a dificuldade para obtenção de licença ambiental, o alto custo de produção e a sazonalidade da produção. Sistemas de Informação Geográfica (SIG) mostram-se como ferramentas capazes de contribuir para a solução desses entraves, já que permitem a captura, análise e visualização de dados referenciados geograficamente. Este trabalho objetiva apresentar um exemplo de aplicação de uma ferramenta de geotecnologia com potencial de agregar informações e contribuir com o desenvolvimento do setor. A seguir, são apresentados e discutidos dados de produção georreferenciados de quatro municípios catarinenses, bem como as principais indústrias de apoio ao setor.
\end{abstract}

Termos para indexação: SIG; Peixe; Cadeia produtiva; Geotecnologia.

\section{Development of a pilot system of geographic data for the management of continental fish farming in Santa Catarina, Brazil}

Abstract - In spite of its recent constant growth, fish farming in the state of Santa Catarina, Brazil, develops in the midst of serious impairments, such as the disorder in the production chain, difficulties in obtaining environmental license, high production costs and seasonality. Geographic Information Systems (GIS) are tools that contribute towards the solution of such impairments since they allow the capture, analysis and visualization of geographically referenced data. Current study aims at providing an example of the application of a geo-technological tool with the potential of aggregating knowledge and contributing to the state policies on the segment. Geo-referenced data on production of four municipalities in the state of Santa Catarina and the sector's main industries are discussed.

Index terms: GIS; Fish; Production chain; Geotechnology.

\section{Introdução}

Segundo a Associação Brasileira de Piscicultura (PeixeBR), a piscicultura continental é a atividade aquícola com maior representatividade em termos de produção, alcançando $758 \mathrm{mil}$ toneladas em 2019 (PeixeBR, 2020). No ranking dos principais estados, Santa Catarina é apontado como o quarto maior produtor nacional de peixes de cultivo, respondendo por 50.200 toneladas de produção de pescado em 2019 (PeixeBR, 2020).

Apesar do cenário otimista de crescimento, a piscicultura continental em Santa Catarina se desenvolve em meio a diversas dificuldades e em um perfil geográfico bastante diverso. Entre os obstáculos ao crescimento da piscicultura, destaca-se a desorganização da cadeia produtiva, o alto custo e a sazonalidade da produção.

Neste contexto, Sistemas de Informação Geográfica (SIGs) mostram-se como ferramentas capazes de contribuir para o melhor entendimento da cadeia produtiva como um todo. SIGs são sistemas computacionais que permitem a captura, o armazenamento, a análise e a visualização de dados referenciados geograficamente. Por meio dele, é possível entender melhor as relações, os padrões e as tendências de determinado setor justamente por relacionar informações descritivas de um fenômeno geográfico a partir de sua localização e vice-versa.

Em vista disso, objetivou-se o desenvolvimento de um SIG piloto para a piscicultura continental comercial catarinense que incorporasse um banco de dados descritivos e geográficos relacionados à atividade em quatro municípios de diferentes regiões do estado: Massaranduba (litoral norte), Grão-Pará (sul) e Belmonte e Barra Bonita (oeste).

\section{Metodologia}

Durante o ano de 2018, o serviço de extensão levantou dados descritivos e de localização geográfica das pisciculturas comerciais dos municípios que foram objeto do estudo. Nestas propriedades rurais, os extensionistas marcavam pontos sobre os corpos d'água utilizando o software Google Earth. Posteriormente, estes pontos foram transformados em polígonos

2 Engenheiro-agrônomo, Epagri/Cedap, CEP 88034-901, Florianópolis, SC, e-mail: novaes@epagri.sc.gov.br

${ }^{3}$ Engenheiro de Aquicultura, Epagri/Cedap, CEP 88318-112, Itajaí, SC, e-mail: brunosilva@epagri.sc.gov.br 
que apresentavam a forma dos viveiros de peixes, utilizando-se imagens de satélite de alta resolução (Google satellite layer) e o software Quantum GIS. Este software também foi utilizado para calcular a área dos polígonos demarcados e para associar as informações de localização geográfica aos dados descritivos levantados. Para apresentação final dos resultados, os dados foram trabalhados no software ArcGis, modalidade Developer.

Paralelamente, foram georreferenciados os principais elos da cadeia produtiva, como pesque-pagues, produtores de alevinos, fábricas produtoras de equipamentos e kits colorimétricos de análise de água, fábricas de ração, estabelecimentos de processamento de pescado com SIF e fábricas produtoras de aeradores e/ou alimentadores automáticos distribuídos ao longo de todo o território catarinense. A lista da relação dos estabelecimentos para abate, entreposto-frigorífico e beneficiamento de pescado em geral com serviço de inspeção federal (SIF) foi adquirida por meio de acesso ao site do Ministério da Agricultura, Pecuária e Abastecimento (Mapa), ao passo que os outros dados acima listados foram obtidos por meio de cadastros prévios provenientes de pesquisas anteriores disponibilizados pela Epagri e busca direta na internet.

\section{Principais resultados}

No total foram cadastradas 110 pisciculturas comerciais (isto é, aquelas que destinam a sua produção à comercialização). Os dados de produção estão apresentados na Tabela 1.

A produção comercial nos municípios estudados utilizou majoritariamente a espécie tilápia-do-nilo, corroborando os dados estatísticos da aquicultura provenientes do InfoAgro, que apontam que a produção de tilápia em 2018 em Santa Catarina representou $88,21 \%$ da produção total comercial. Nos municípios de Belmonte, Barra Bonita e GrãoPará a produção comercial concentrouse totalmente $(100 \%)$ na tilápia-do-nilo, mas no município de Massaranduba registrou-se uma pequena produção (11.385kg; $0,73 \%$ do total produzido no município) de outras espécies, prin-
Tabela 1. Dados descritivos e índices produtivos levantados nos municípios avaliados, provenientes de pisciculturas comerciais em 2018

Table 1. Descriptive data and production indexes on commercial fish farms in the evaluated municipalities, in 2018

\begin{tabular}{|c|c|c|c|c|}
\hline & $\begin{array}{l}\text { Belmonte } \\
\text { Oeste }\end{array}$ & $\begin{array}{c}\text { Barra } \\
\text { Bonita } \\
\text { Oeste }\end{array}$ & $\begin{array}{l}\text { Massaran- } \\
\text { duba } \\
\text { Litoral Norte }\end{array}$ & $\begin{array}{l}\text { Grão-Pará } \\
\text { Sul }\end{array}$ \\
\hline $\begin{array}{l}\text { Número de produtores } \\
\text { comerciais }\end{array}$ & 2 & 2 & 52 & 54 \\
\hline $\begin{array}{l}\text { Produtividade média } \\
\qquad\left(\mathrm{t} \mathrm{ha}^{-1}\right)\end{array}$ & $\begin{array}{c}16,49 \\
(14,10- \\
21,85)^{*}\end{array}$ & $\begin{array}{c}20,27 \\
(20,12- \\
20,92)\end{array}$ & $\begin{array}{c}20,09 \\
(1,76- \\
115,17)\end{array}$ & $\begin{array}{c}15,22 \\
(3,20- \\
52,2)\end{array}$ \\
\hline Produção total (t) & 20,72 & 48,00 & $1.432,66$ & 860,25 \\
\hline Área total alagada (ha) & 1,26 & 2,37 & 71,31 & 56,5 \\
\hline $\begin{array}{l}\text { Área média alagada/ } \\
\text { produtor (ha) }\end{array}$ & 0,63 & 1,18 & 1,37 & 1,05 \\
\hline Área média do viveiro $\left(\mathrm{m}^{2}\right)$ & 1.794 & 2.153 & 2.958 & 3.646 \\
\hline $\begin{array}{l}\text { Número médio de viveiros } \\
\text { por produtor }\end{array}$ & $\begin{array}{c}3,5 \\
(2-5)\end{array}$ & $\begin{array}{c}5,5 \\
(3-8)\end{array}$ & $\begin{array}{c}4,6 \\
(1-16)\end{array}$ & $\begin{array}{c}2,9 \\
(1-9)\end{array}$ \\
\hline $\begin{array}{l}\text { Peso médio final na } \\
\text { despesca (g) }\end{array}$ & $\begin{array}{c}802,5 \\
(765-840)\end{array}$ & $\begin{array}{c}775 \\
(750-800)\end{array}$ & $\begin{array}{c}878,94 \\
(550-1200)\end{array}$ & $\begin{array}{c}733,82 \\
(550- \\
1000)\end{array}$ \\
\hline \multicolumn{5}{|c|}{ Licença ambiental (\%) } \\
\hline $\operatorname{Sim}$ & 50 & 50 & 19,64 & 50,91 \\
\hline Não & 50 & 50 & 44,64 & 49,09 \\
\hline Não informado & - & - & 35,71 & - \\
\hline \multicolumn{5}{|c|}{ Origem dos alevinos (município) (\%) } \\
\hline Ilhota (SC) & 50 & - & 48,21 & 16,36 \\
\hline Pomerode (SC) & - & - & 35,72 & - \\
\hline São Ludgero (SC) & - & - & - & 10,91 \\
\hline Rolândia (PR) & - & - & 8,93 & - \\
\hline Braço do Norte (SC) & - & - & - & 58,18 \\
\hline São Miguel do Oeste (SC) & - & 50 & - & - \\
\hline Toledo (PR) & 50 & 50 & - & - \\
\hline Outros & - & - & 7,14 & 14,55 \\
\hline \multicolumn{5}{|c|}{ Destino final da produção (\%) } \\
\hline Frigorífico (SC) & 100 & 100 & 44,64 & 92,73 \\
\hline Pesque-pague (SC) & - & - & 17,86 & 1,82 \\
\hline Frig. e Pesque-pague (SC) & - & - & 16,07 & - \\
\hline Pesque-pague (PR) & - & - & 8,93 & - \\
\hline Pesque-pague (SP) & - & - & 7,14 & - \\
\hline Não informado & - & - & 5,36 & 5,45 \\
\hline
\end{tabular}

Números entre parênteses correspondem aos valores mínimo e máximo. 
cipalmente de jundiás e carpas, que foram, em sua maioria, destinadas aos pesque-pagues.

Apesar de os valores de produtividade terem variado consideravelmente entre cada piscicultura analisada, a média permaneceu similar entre os municípios estudados, variando de 15 a 20t/ ha (Tabela 1). Ainda que esse valor, segundo os autores, possa ser melhorado por meio da implementação de técnicas adequadas e boas práticas de manejo, o mesmo já aumentou substancialmente considerando os últimos quatro anos, período em que a média de produtividade comercial no Estado era de apenas 7,4 toneladas por hectare, segundo os dados da Epagri (SILVA et al., 2017).

O peso médio final da tilápia na despesca também variou entre as pisciculturas e os municípios estudados (Tabela 1). Contudo, a média $( \pm 800 \mathrm{~g})$ sugere um aumento no tamanho do peixe comercializado ao longo dos últimos dez anos, tendo este aumento ocorrido em virtude de tendências do mercado e preferências do consumidor final. Com relação à cadeia produtiva, foram cadastrados 235 pesque-pagues, 21 produtores de alevinos, 7 fábricas de equipamentos e kits colorimétricos de análise de água, 7 fábricas de ração, 76 estabelecimentos de processamento de pescado com SIF e 8 fábricas de aeradores e alimentadores automáticos em todo o Estado. Dos pesque-pagues cadastrados, a maioria se concentra nas regiões de Itajaí/Blumenau, Joinville e Rio do Sul, onde diversas cidades oferecem aos moradores e visitantes a experiência da pescaria, com forte apelo cultural. É provável que, em virtude disso, o município de Massaranduba (litoral norte) destine uma parte expressiva da sua produção aos pesque-pagues, diferentemente dos outros municípios analisados, cujo destino na quase totalidade são os frigoríficos. No que se refere aos estabelecimentos de beneficiamento de pescado com SIF, quase $90 \%$ deles estão localizados no litoral do Estado, incluindo as regiões de Itajaí/Blumenau $(61,8 \%)$, Florianópolis $(15,7 \%)$ e Tubarão (11,8\%). As fábricas de aeradores e/ ou alimentadores automáticos, por sua vez, estão todas localizadas nas regiões de Joinville e Itajaí/Blumenau, onde, não por acaso, também está localizado

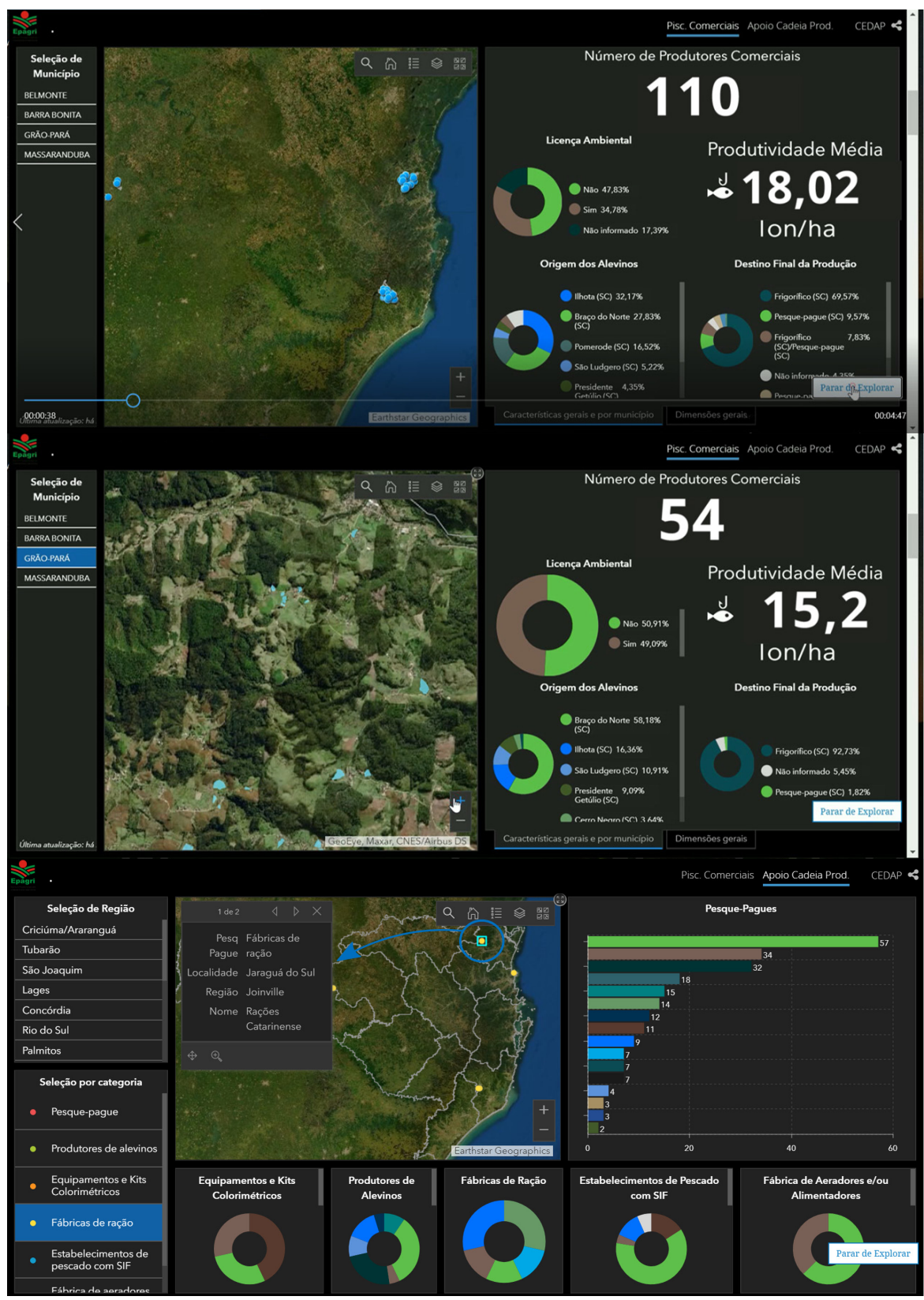

Figura 1. Apresentação do sistema de informações geográficas - projeto-piloto - da piscicultura continental catarinense. A. Mapa evidenciando as pisciculturas cadastradas representadas por pontos nos municípios de interesse. B. Aproximando-se dos pontos, estes dão espaço às unidades produtoras de peixes (destacadas em azul). C. Clicandose em cima de um ponto ou unidade produtora de peixes, tem-se acesso a informações descritivas da atividade.

Figure 1. Geographic information system - pilot plan - of fish farms in the state of Santa Catarina, Brazil. A. Map with enrolled fish farms by dots on the municipality map. $B$. Production units, close to the dots, in blue. C. Clicking on the dot or on fish producing unit, you obtain the units description data.

o principal polo metalmecânico de Santa Catarina.

Dos quatro municípios analisados neste estudo, os dois maiores produtores (Massaranduba e Grão-Pará) estão próximos de importantes elos da cadeia produtiva, tais como produtores de alevinos, fábricas de ração e de ae- radores e/ou alimentadores automáticos. Provavelmente, essa proximidade favorece a ampliação da piscicultura e a consolidação desses municípios como importantes centros produtores no Estado. Quanto à origem dos alevinos, foi possível perceber uma forte tendência de escolha por produtores de alevinos 
próximos (em média, 100km de distância) dos municípios em estudo (Tabela 1), facilitando a logística de transporte.

A visualização dos dados é apresentada na Figura 1. Por meio dos painéis criados, o usuário pode interagir com os dados, acessando a informação de seu interesse. No mapa, cada piscicultura é representada por um ponto (Figura 1A) e, na medida em que se aproxima do mesmo, dá-se espaço às unidades produtoras (viveiro, tanque ou açude) (Figura 1B). Clicando-se na unidade produtora em destaque, é possível acessar informações descritivas (contextuais) associadas a ela (Figura 1C). Ao lado do mapa, gráficos referentes aos dados coletados por município são apresentados e atualizados simultaneamente à pesquisa do usuário (Figura 1).

\section{Potenciais para}

\section{preenchimento de lacunas técnicas e geográficas}

Os autores creem no potencial da ferramenta para auxiliar a adequação dos locais e do zoneamento, o planejamento estratégico para o desenvolvimento, ampliar a capacidade de integrar dados e informações dispersas, otimizar o suporte tecnológico em instituições públicas e privadas e monitorar as atividades produtivas, além de dar apoio ao direcionamento e à melhoria da eficácia de políticas públicas.

\section{Considerações finais}

O sistema integra a localização geográfica às informações de interesse do setor produtivo da piscicultura. Tratase de uma ferramenta com potencial de auxiliar a gestão e a estruturação das cadeias de suprimento e a comercialização da produção útil na tomada de decisões que objetivem a melhoria de resultados dos empreendedores do setor e o aumento da competitividade da atividade.

\section{Agradecimentos}

Os autores agradecem aos profissio- nais Luiz F. N. Vianna, Fabiano Muller Silva, Everton G. Della Giustina, Darci Pitton Filho, Luiz R. M. Vicente, Osvaldo Ruppel, Cintia Regina Veiga e Tatiane Carine da Silva pelo apoio técnico e operacional.

\section{Referências}

ASSOCIAÇÃO BRASILEIRA DA PISCICULTURA (PEIXEBR). Anuário PeixeBR da piscicultura 2020. Disponível em: www.peixebr.com.br. Acesso em: 26 jun. 2020.

SECRETARIA DE ESTADO DA AGRICULTURA E DA PESCA. Mapa interativo de produção de peixes. Disponível em: https://app.powerbi. com/view?r=eyJrljoiMTc1MTg4YzYtZGMONCO0M2IOLWE3N2EtYWY10GYYZWY5YTCZIi widCI6ImExN2QwM2ZjLTRiYWMtNGI2OC1iZDY4LWUzOTYZYTJIYzRINiJ9. Acesso em: 31 out. 2019.

SILVA, B.C.; GIUSTINA, E.G.D.; MARCHIORI, N.C.; MASSAGO, H. SILVA, F.M. Desempenho produtivo da piscicultura catarinense em 2015. Florianópolis, SC: Epagri, 2017, 17p. (Documentos, 268).

\section{Não deixe sua consciência escorrer pelo ralo: preserve a água e evite o desperdício.}

